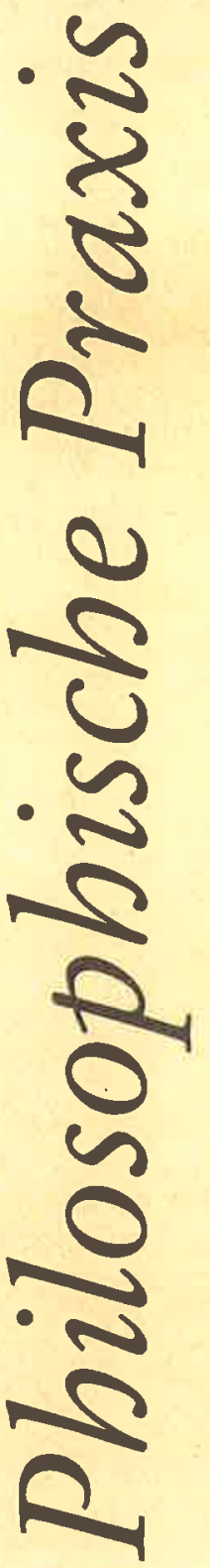

Norbert Hinske:

Fata ducunt

Patrick Neubaner:

Zur Rehabilitation des Unbewußten

Andreas Dorschel:

Über die Intentionalität von Emotionen

Gerhard Stamer:

Irrationalität und kritisches Bewußtsein

Pbilip Whyte:

Did the Enlightenment just happen to men?

Anja Overdiek:

Ein Mann sieht vor lauter Welle das Wasser nicht

Bericht:

Zur Lage der Philosophischen Praxis

Die Seele in der Moderne. Therapie, Seelsorge, Philosophische Praxis 


\section{ÜBER DIE INTENTIONALITÄT VON EMOTIONEN}

von Andreas Dorschel

\section{DER BEGRIFF DES INTENTIONALEN OBJEKTS EINER EMOTION}

Ist jemand erfreut, oder traurig, oder in Furcht, so lassen sich stets Fragen stellen wie: erfreut worüber? traurig worüber? in Furcht wovor? Diese Eigenschaft von Emotionen, daß in bezug auf sie solche Fragen möglich und sinnvoll sind, wird Intentionalität ${ }^{1}$ genannt, d.h.: ein emotionaler Zustand hat einen Inhalt, oder: ist gerichtet auf ein Objekt. Diese Rede vom 'Objekt' einer Emotion ist abgeleitet vom grammatischen $\mathrm{Be}$ griff des Objekts eines transitiven Verbs: das Objekt der Furcht ist das, was einer fürchtet, das Objekt der Liebe das, was einer liebt. (Und pedantischerweise kann angemerkt werden, daß, wie sich bereits andeutete, die Sprache für viele Emotionen die Form des Präpositionalobjekts - das Objekt des Stolzes ist das, worauf man stolz ist, das Objekt der Empörung das, worüber man empört ist usw. - bevorzugt, ohne daß daraus Aufschlußreiches über den Charakter dieser Emotionen im Unterschied zu den mit einfachem Akkusativobjekt ausgedrückten zu folgern wäre ${ }^{2}$. Die besagte Ausdrucksweise, und insbesondere die Rede von einem Objekt, ist ebenso unverzichtbar, wie sie erläuterungsbedürftig ist. Sie scheint nämlich nahezulegen, das Objekt, insofern beansprucht ist, es handele sich um ein solches, müsse existieren ${ }^{3}$. Dies ist jedoch unzutreffend. Das - wie man erläuternd hinzufügt: intentionale Objekt kann bloße Vorstellung bleiben oder gar pure Einbildung sein. Jemand kann erfreut sein über etwas, von dem er nur glaubt, es sei der Fall, obwohl es nicht der Fall ist. Jemand kann auf Regen hoffen und sein himmlisches Idol verehren, auch wenn der Regen ausbleibt und Gott ein Produkt seiner Phantasie ist. Jemand kann Angst haben vor Mäusen unter seinem Bett, auch wenn dort keine sind. Und er hat dann nicht etwa Angst vor seiner Überzengung, daß Mäuse unter dem Bett sind.

Wenn sich jemand vor dem Weihnachtsmann fürchtet, folgt aus der Feststellung, daß es den Weihnachtsmann nicht gibt, keineswegs, daß die Furcht eingebildete Furcht ist. Das Subjekt der Furcht fürchtet sich vielmehr ungeachtet des besagten Umstands wirklich vor dem Weihnachtsmann. Die Konsequenz, die sich aus der Auffassung strikter Nominalisten ergäbe, die in der Rede von intentionalen Objekten die Annahme überflüssiger Wesenheiten impliziert sehen und daher insistieren, es könne einer nicht wirklich fürchten, was nicht existiere: die Konsequenz nämlich, daß niemand je wirklich Gespenster und Kobolde gefürchtet hätte, ist durchaus abstruser als dic vorgebliche Abstrusität, die jene Auffassung zu verhindern behauptet.

Einerseits ist also hinsichtlich der Existenz des Objekts Irrtum möglich. Andererseits aber kann das Objekt, wenn es existiert, doch in seinen Eigenschaften gründlich verkannt werden. Ein Papiertiger kann Furcht verbreiten, deren Inhalt (intentionales Objekt) nicht ein papierener, sondern ein lebendiger Tiger ist. Man kann auch sagen: reales und intentionales Objekt brauchen sich nicht zu decken. In diesem Zusammenhang ist es von Belang, daß zwischen einer Überzeugung und einer Emotion ein Verhältnis der Voraussetzung bestehen kann. Um, beispielshalber, stolz zu sein auf etwas, muß man dieses Etwas als in irgendeiner Weise mit sich verbunden betrachten. Dabei handelt es sich aber um keine Restriktion dessen, was als reales Objekt des Stolzes in Frage kommt. Was vorliegt, ist vielmehr eine Restriktion, die sich auf die Sicht des
Subjekts der Emotion auf das Objekt bezieht: auf die Art und Weise, wie es dieses betrachtet. Daß nur getrocknet werden kann, was naß, oder wenigstens feucht ist, formuliert eine Einschränkung für die möglichen Objekte des Trocknens. Daß man nur auf das stolz sein kann, was in irgendeiner Weise einem selber zugehört, formuliert eine Einschränkung für die möglichen Objekte des Stolzes. Aber während jene Behauptung richtig war, ist diese falsch. Berichtigt man sie jedoch dahingehend, daß einer nur auf das stolz sein kann, wovon er glaubt, daß es ihm in irgendeiner Weise zugehört, so formuliert man keine Einschränkung der möglichen Objekte des Stolzes, sondern der Überzeugungen des stolzen Subjekts. Es gibt keine Objekte, auf die einer nicht, hinreichend phantastische Überzeugungen vorausgesetzt, stolz sein könnte. Hält einer sich für den göttlichen Schöpfer des Planeten Neptun, so dürfte es ihn keine Mühe kosten, auf dessen Äquatordurchmesser stolz zu sein. Wenn die Beziehungen von Emotionen zu ihren Objekten analysiert werden, so ist dies, wenigstens solange die Analyse eine korrekte ist, mithin nie so zu verstehen, als würde jeweils eine Anzahl realer Gegenstände aus der Gesamtheit des Wirklichen herausgenommen, und bestimmten Typen von Emotionen, als deren mögliche Objekte, gegenübergestellt.

Noch in einer anderen Hinsicht ist die Rede vom 'Objekt' einer Emotion erläuterungsbedürftig. Es fallen unter diesen Titel nämlich nicht lediglich Gegenstände, sondern auch Ereignisse (jemand kann außer sich sein vor Freude über die Vorgänge in Rußland, empört darüber, daß sein Name auf die Schwarze Liste gesetzt wurde, oder entsetzt über einen Unfall), Zustände, Attribute oder Qualitäten von Dingen (jemand kann betrübt sein über den schlechten Zustand seines Hauses) oder Personen (jemand kann beeindruckt sein von der Eloquenz eines anderen, oder neidisch auf seinen Reichtum), sowie ihr materieller oder ideeller Ausstoß (jemand kann ärgerlich sein über den Qualm der Chemiefabrik nebenan, oder beunruhigt über die Äußerungen des Verteidigungsministers), Verhältnisse und Beziehungen (jemand kann eifersüchtig sein auf die Freundschaft seiner Frau mit einem anderen Mann), Handlungen und Verhaltensweisen (jemand kann beschämt sein über ein sexuelles Vergnügen, dem er nachgegangen ist, oder peinlich berührt durch jemandes schlechte Manieren). Emotionen können auch Emotionen zu ihrem Gegenstand haben: jemand kann stolz darauf sein, daß er traurig ist, oder traurig darüber, daß er stolz ist. Diese Beziehung subjektiver Haltungen auf subjektive Haltungen ist prinzipiell indefinit; es kann sich jemand dafür schämen, daß er stolz darüber ist, daß er traurig ist - usf. Ferner kann einer aber auch stolz darauf sein, daß er stolz ist, traurig daruber, daß er traurig ist, oder Angst vor der Angst haben. So kann ein wesentliches Moment des Denkens - Reflexion - in die angeblich gedankenlosen Emotionen eintreten. (Sofern dies Moment durch die Erinnerung vermittelt ist, entsteht die Möglichkeit der Sentimentalität. Erinnerung, einerseits das Herstellen einer Beziehung zu etwas, ist andererseits dessen Distanzierung. Qua Erinnertes ist es als vergangen ausgewiesen. Die Erinnerung macht es zu einem nicht wiedererlebbaren Inhalt, auch wenn zum Beispiel die Erinnerung mit dem Erinnern durchlittener Trauer eine Emotion gleicher Qualität erzeugen kann; diese ist eine neue, anläßlich der Erinnerung entstandene, sentimentale Emotion.) 


\section{DIE UNTERSCHEIDUNG VON OBJEKT UND URSACHE EINER EMOTION}

Auf den ersten Blick nimmt es sich sinnvoll aus, bei der Frage nach dem Objekt zwischen Gefühlen und Emotionen zu differenzieren. Ja, es scheint sich anzubieten, Gefühle und Emotionen gerade anhand des Kriteriums zu unterscheiden, daß das Objekt der letzteren, nicht aber der ersteren immer ein bestimmter einzelner Gegenstand (ein bestimmtes einzelnes Ereignis, Verhältnis usw.) sein müsse. Hunger, genauer: das Hungergefühl ${ }^{4}$ ist zwar stets auf Nahrung als sein Objekt gerichtet. Aber es ist möglich, hungrig zu sein, ohne auf etwas Bestimmtes hungrig zu sein, in einem Sinne, in dem es nicht möglich ist, beschämt zu sein, ohne über etwas Bestimmtes beschämt zu sein. Indessen scheint, daß Emotionen auf einzelne Objekte gerichtet sind, nicht allgemein zuzutreffen. Selbst wenn einer nicht beschämt sein kann, ohne über einen bestimmten cinzelnen Vorfall beschämt zu sein, so kann er doch Furcht haben, ohne vor einem bestimmten einzelnen Gegenstand Furcht zu haben. So kann einer Furcht vor Feuer haben, auch wenn es kein bestimmtes einzelnes Feuer gibt, vor dem er sich fürchtet. Solche Furcht verhält sich ganz analog dem Hunger, der sich auf Nahrung bezieht, und nicht auf einen bestimmten Apfel beziehen muß ${ }^{5}$. - Man könnte noch versuchen, die in Frage stehende These mit folgendem Unterschicd zu stützen: Es ist möglich, Schmerzen zu haben, ohne zu wissen, was einen verletzt, in einem Sinne, in dem es nicht möglich ist, sich zu freuen, ohne zu wissen, was einen freut. Aber dieses Argument ist schief. Was einen verletzt, ist die Ursache des eigenen Schmerzes, und diese kann einem zwar unbekannt sein, - ebenso kann einem aber die Ursache der eigenen Freude unbekannt sein.

Die Unterscheidung zwischen dem Objekt einer Emotion und der Ursache einer Emotion ${ }^{6}$ ist, ungeachtet der Vagheit und Mehrdeutigkeit beider Ausdrücke, jedenfalls die zwischen dem, was einer fürchtet, liebt usw., und dem, warum er sich fürchtet, liebt usw. (Die Warum-Frage ist freilich ihrerseits ambig, und kann sich, statt auf die Ursachen, auch auf die Gründe von Emotionen beziehen.) Es gibt viele Fälle, in denen es naheliegt, das Objekt der Emotion als ihre Ursache anzusehen, zum Beispiel: 'Ich war ärgerlich auf sie', oder 'Thr Verhalten brachte mich in Verlegenheit'. So ist nicht ohne weiteres klar, wie die Differenzierung zwischen der Ursache und dem Objekt zu begründen ist. Ein Argument, das ihre Nötigkeit zu erweisen scheint, lautet folgendermaßen: Das Objekt von Stolz und Scham ist das eigene Selbst und seine Äußerungen. Jemand kann, selbst zur gleichen Zeit, stolz auf sich sein und sich seiner schämen; er kann über ein und das selbe grandios infame Verhalten, das er an den Tag gelegt hat, in diese ganz entgegengesetzten Emotionen geraten. Wäre das eigene Selbst, das das Objekt dieser Emotionen ist, zugleich deren Ursache, so wäre das mit dem Prinzip unvereinbar, daß gleiche Ursachen gleiche Wirkungen, verschiedene Ursachen verschiedene Wirkungen, entgegengesetzte Ursachen entgegengesetzte Wirkungen zeitigen? ${ }^{7}$. Das Argument ist jedoch angreifbar, und zwar sowohl auf der Grundlage dieses Prinzips, wie auch in Zurückweisung desselben. Zum einen kann eingewandt werden, daß das Objekt - das Selbst bzw. das grandios infame Verhalten, dem es seine Umgebung ausgesetzt hat - schon in sich entgegengesetzt gewesen sei, und also auch die Ursache von Entgegengesetztem sein könne. Zum anderen kommt es durchaus vor, daß eine Ursache zwei und mehr sehr verschiedene Konsequenzen nach sich zieht.

Gleichwohl ist die Unterscheidung zwischen Objekt und Ursache von Emotionen notwendig und richtig. Denn Aussa- gen über die Ursache und Aussagen über das Objekt einer Emotion bedeuten Verschiedenes. Die Feststellung der Ursache einer Emotion braucht nichts über ihr Objekt zu besagen. Wenn jemand feststellt, daß seine Depression durch zu viel Aspirin verursacht war, so muß dies keineswegs heißen, daß er deprimiert war über seinen übermäßigen Aspirinkonsum, d.h. daß dieser den Inhalt seiner Depression ausmachte. Die Ursache von jemandes Ärger mag zu wenig Schlaf oder zu viel Kaffee sein. Aber er braucht, damit diese Aussage wahr ist, keineswegs ärgerlich zu sein über seinen Schlafmangel oder ̈̈ber die Hyperstimulation durch Coffein. Freilich belegt dieses Argument, wie auch das folgende, daß Ursache und Objekt einer Emotion nicht zusammenfallen müssen, nicht aber, daß sie nicht zusammenfallen können. Wenn jemand über seinen Gesundheitszustand deprimicrt ist, so kann dieser sowohl die Ursache wie auch das Objekt seiner Depression sein.

Ist das Objekt einer Emotion ein künftiges Ereignis - etwa, wenn jemandem vor dem III. Weltkrieg graut, oder wenn einer auf den Sieg der Faschisten bei der nächsten Wahl hofft -, so kann es ipso facto nicht die Ursache dieser Emotion sein, weil Ereignisse oder Zustände in der Zukunft keine Ereignisse oder Zustände in Gegenwart oder Vergangenheit verursachen. Daß jemand eine Explosion fürchtet, besagt, er fürchte, daß es eine geben wird. Es braucht keine vergangene Explosion gegeben zu haben, die die Ursache, oder auch nur eine Ursache seiner Furcht war ${ }^{8}$. Sollte es aber eine vergangene Explosion gegeben haben, so ist nicht sie das, was der Betreffende fürchtet: sie ist nicht das Objekt seiner Furcht. Und wenn irgendeine Explosion in der Zukunft geschehen sollte, die seiner Furcht Recht gibt, weil sie wahr macht, was er befürchtet hat, so wird diese Explosion ex hypothesi, als künftige, nicht die Ursache, oder auch nur eine Ursache, seiner vorausgegangenen Furcht gewesen sein. Denn, abgesehen von dem bereits angeführten Grund (was Wirkung einer Ursache ist, kann dieser nicht zeitlich vorausgehen), war das, was der Betreffende befürchtet hat (das sogenannte Objekt seiner Furcht) nicht diese Explosion, das konkrete Ereignis, das seine Furcht bestätigt hat, sondern irgendeine Explosion innerhalb bestimmter typischer, aber vager Parameter, zum Beispiel eine Explosion im linken Reaktor des Kraftwerks, verursacht durch Defekte an den zu lange Zeit eingesetzten Brennstäben, innerhalb der nächsten Wochen. Es gibt kein wirkliches vergangenes, gegenwärtiges, oder künftiges Ereignis, das identisch mit dem Objekt seiner Furcht wäre. Gleichwohl ist das, was der Betreffende fürchtete, abstrahierbar, theoretisch unterscheidbar von der Furcht, und in diesem anspruchslosen Sinne wird der Terminus 'Objekt' im vorliegenden Zusammenhang verwendet. Freilich bleibt mit der Betrachtung dieses Falles einer Emotion für andere Fälle die Koinzidenz von Ursache und Objekt als Möglichkeit offen. Wie bemerkt, können beide zusammenfallen, und es ließe sich unterscheiden zwischen Emotionen, deren Objekte ihre Ursachen sind, und Emotionen, deren Objekte nicht ihre Ursachen sind ${ }^{9}$.

\section{DAS VERHÄLTNIS VON OBJEKT UND URSACHE EINER EMOTION UND DAS PRINZIP DER PSYCHOTHERAPIE}

Das Verhältnis von Objekt und Ursache ist für die Beurteilung von Emotionen als vernünftig oder unvernünftig von einigem Belang. Im Falle der auf die Vergangenheit und Gegenwart bezogenen Emotionen ist die Koinzidenz von Objekt und Ursache eine Rationalitätsanforderung an Emotionen. Das heißt: Es kann für sie nicht zugleich die Divergenz von Objekt und Ursache einer Emotion und ihre Vernünftigkeit behauptet werden. Das besagt nicht, daß jemand nur ärgerlich 
sein kann, solange er glaubt, daß das, was seinen Ärger verursacht hat, zugleich das ist, worüber er sich ärgert. Aber glaubt er dies nicht, so kennzeichnet er seine Emotion mit der Feststellung der Divergenz von Ursache und Objekt implizit als unvernünftig. Und darüberhinaus kann er das, was er zuvor für das Objekt der Emotion gehalten haben mag, nicht mehr weiter als Objekt der Emotion behaupten. Das Wissen um die von dem Objekt der Emotion verschiedene Ursache der Emotion hat die Tendenz, die Emotion selbst zu untergraben. Man kann, sobald man entdeckt hat, daß das bisherige Objekt des eigenen Gereiztseins nicht dessen Ursache ist, nicht mehr über jenes gereizt sein, mag man im übrigen auch reizbar bleiben. Es ist ohne Sinn, von sich zu sagen 'Ich bin ärgerlich darüber, daß sie meinen Wagen gestohlen hat, aber sie hat ihn nicht gestohlen und mein Ärger kommt nur daher, daß ich zu viel Kaffee getrunken habe'. Ist das letztere eingeräumt, so ist zugleich zugestanden, daß die Emotion zu verschwinden hätte (was freilich ge- oder mißlingen kann). Das letztere mag allerdings eine Eigentümlichkeit dieser gegen jemand gerichteten Emotion sein; wer irgendeine Laune oder Lappalie als die von dem Objekt seiner Freude differente Ursache derselben feststellt, räumt damit schwerlich ein, daß seine Freude aufzuhören hätte. Gleichwohl ist seine Freude ungerechtfertigt, und taugt nicht als Argument für oder gegen irgendetwas, während Emotionen sonst, wenn, weil und insofern sie begründet sind, (entgegen ihrer notorischen Perhorreszierung in Argumentationen) durchaus als Argument taugen können. Begründeter Ärger ist nicht einfach abzuweisen als Privatangelegenheit des ärgerlichen Subjekts, das mit seinem Innenleben therapeutisch ins Reine zu kommen hätte, sondern durchaus ein Argument, den ärgerlichen Sachverhalt abzustellen. Daß aber eine Emotion begründet ist, ist eine Eigenschaft, die im Falle der auf die Vergangenheit und Gegenwart bezogenen Emotionen zur (notwendigen, wenngleich nicht hinreichenden) Bedingung hat, daß der selbe Sachverhalt Ursache und Objekt der Emotion ist. $\mathrm{Ob}$ es sich im einzelnen Fall jeweils so verhält oder nicht, ist eine Frage, von der immer da abgesehen wird, wo Affekte, Emotionen, Leidenschaften wie physische Funktionsstörungen vorgestellt, oder gar ihnen gleichgesetzt werden was philosophisch durchaus Tradition hat ${ }^{10}$.

Praktisch relevanter als letztere ist jener Standpunkt psychologischer Therapie, der von vornherein vom Objekt der Emotion abstrahiert, und sich einer ihn konstituierenden methodischen Vorentscheidung folgend exklusiv auf die Suche nach der Ursache der Emotion im Subjekt begibt. Als Therapie scheidet darum eines von vornherein aus: die Beseitigung des Grundes etwa des Ärgers. Die Frage nach der Begründetheit der Emotion wird durch die ihr äußerliche Unterscheidung zwischen "gewöhnlichem« und »ungewöhnliche[m] Mißmut «11 ersetzt. Der Psychologe nähert sich zwar dem Seelenleben im ganzen mit der Attitüde des Mediziners - nach Freud ist »der normale Affekt « vergleichbar »dem Ausdruck einer generellen, zur Erbschaft gewordenen Hysterie « ${ }^{12}$-, läßt sich dann aber das Kriterium der Behandlungsbedürftigkeit darin vorgeben, ob jemand störend auffällt oder nicht. Der in besagter Unterscheidung zwischen "gewöhnlichem « und "ungewöhnlichem Mißmut« in Anschlag gebrachte Maßstab bedeutet in zweifacher Hinsicht eine Abstraktion: den, der ihn anlegt, interessiert weder ein - subjektives oder gesellschaftliches - Wofür des Funktionierens, das er dem in "gewöhnlicher "Weise reagierenden, d.b. gesunden Subjekt als dessen normale und hauptsächliche Eigenschaft nachsagt, noch will er etwas davon wissen, welche - richtigen oder verkehrten Gründe jemand für sein Verhalten hat, was also den Inhalt seines Willens ausmacht. Die letztere Abstraktion erstreckt sich auch auf die Emotion: von deren Grund ist abgesehen, inso- fern Gegenstand nur die subjektive Reaktion selber ist. Dies präjudiziert, der Patient leide am Ärger und nicht an seiner Lage; folgerichtig erscheint Ärger als Problem dessen, der sich ärgert: er ist frustriert, er hat Probleme, er muß mit sich selbst zurechtkommen. Die Auskunft, er sei frustriert, will so viel sagen wie: er habe zum Gegenstand seines Ärgers noch nicht die richtige, nämlich eine solche Einstellung gefunden, die ihn diesen »[g]elassen «13 ertragen läßt, »Eine Selbstbesänftigung kann also nur gut sein $\aleph^{14}$.

Die getroffene Unterscheidung zwischen dem Objekt und der Ursache einer Emotion - genauer: der Umstand, daß der Glaube an eine vom Objekt der Emotion verschiedene Ursache der Emotion die Tendenz hat, die Emotion selber aufzulösen - vermag zur Erklärung einer recht geläufigen, wenngleich den Psychotherapeuten peinlichen Tatsache beizutragen: der Tatsache, daß trotz der Verschiedenheit der psychotherapeutischen Praktiken und des jeweiligen theoretischen Selbstverständnisses ihrer Vertreter keine nennenswerten Unterschiede in der Wirksamkeit der diversen Therapiearten festzustellen sind. Was die jeweils zugrundeliegenden Annahmen besagen, ist dem Leiden der Klienten offensichtlich gleichgültig. Und so sehr man Grund hat, eine Heilbehandlung verdächtig zu finden, bei der die Diagnose, und mithin die Besonderheit der Krankheit gar keine bestimmte Therapie erforderlich macht, sondern, je nach Vorliebe des Therapeuten, das jeweilige Verfahren immer paßt, so wenig ist dieses Ergebnis unerklärlich: es handelt sich bei den Therapien eben um die Zuordnung einer dem Bewußtsein des Patienten nicht gerade naheliegenden Ursacbe - irgendeiner Ursache - zu, beispielsweise, den für störend erachteten Ängsten. (Vorgreifend formuliert: Wenn der Patient die ihm angebotene Erklärung seiner Ängste, die auf deren Inhalt nicht bezogen ist, übernimmt, so wurde ein therapeutischer Erfolg erzielt.) Werden solche Ängste von jemandem geäußert, so hebt unter den Repräsentanten der Disziplin ein Streit darüber an, ob sie aus dem Verlust des Urvertrauens durch Zugriff der Hebamme, aus allzuvielen Verstärkungen des Angstfaktors im Laufe seines Lebens ("Belohnung von Angstsymptomen «), oder gar daraus zu erklärèn sind, daß er sich im Sinne seiner gottlosen Zivilisation für allmächtig hält und diesen Glauben gefährdet sieht ${ }^{15}$. Bereits die erste Version prägt das Prinzip der Abstraktion vom Inhalt der Angst, ihrem intentionalen Objekt, rein aus. Freud hat die Angst auf den Geburtsakt, die erste Beengung (angustia) im Atmen zurückgeführt ${ }^{16}$. (Die Etymologie fungiert an dieser Stelle - so als ob die Herkunft eines Wortes die Wahrheit eines Gedankens verbürgte - als Argument.) Jede spätere Angst macht nach Freud lediglich dies Urerlebnis von Beklemmung rezent; das angstvolle Reagieren auf Gefahrensituationen soll also darin regressiv und bloß subjektiv sein. "Der Geburtsakt ist « - einer kaum verhüllten Logik des "post hoc, ergo propter hoc « gemäß »das erste Angsterlebnis und somit Quelle und Vorbild des Angstaffekts « ${ }^{17}$, folglich jeder »Angstzustand eine Reproduktion des Geburtstraumas « ${ }^{18}$, so daß Menschen mit den unterschiedlichsten, selbst gegensätzlichsten Zwecken, deren Vereitelung sie ängstigt, in gänzlicher Uniformität stets nur an den Spätfolgen der Entbindung laborieren. Selbst die zweite Erklärungsversion - „Verstärkung des Angstfaktors" -, in der scheinbar die Realität noch vorkommt, teilt mit der ersten »Geburtstrauma - wie der letzten - Gotteskomplex « - die Abstraktion vom Inhalt der für störend erachteten Emotion. Gegenüber dem, wovor er sich ängstigt oder worïber er sich ärgert, sieht sich der Patient auf Instanzen ganz anderer Art verwiesen. Er wird »unterrichtet, was er sehen soll, sonst sieht er es überhaupt nicht « ${ }^{19}$. Mit der Autorität des Fachmanns weist der Therapeut ihn in ein anschauliches Bild vom eigenen »Selbst« ein, dem zufolge dieses das Ambiente eines inneren 
Dramas diverser »Kräfte ${ }^{20}$, Energien und Potenzen ist, welche den Verstand und Willen, den er aufbringt, zur abhängigen Variable herabsetzen. Dieses Bild ist ein Angebot. Sieht sich der Klient veranlaßt, ihm nicht nur Glauben zu schenken, sondern es darüberhinaus zu seinen ganz selbstverständlichen Erfahrungen zu rechnen, und sein Tun und Lassen als Äußerung jener Kräfte sowohl anzusehen als auch, vor allem, methodisch zu handhaben, so hat der Psychologe, wie bereits vorgreifend erwähnt, einen therapeutischen Erfolg erzielt. (Und dies ist schwierig. Denn verlangt wird nichts geringeres als: sich unter eine Determination des eigenen Denkens, Fühlens, Wollens und Handelns so konsequent zu subsumieren, daß man sich als Subjekt dieser Subsumption verleugnet.) Der Erfolg besteht mithin darin, daß das leidende Subjekt seine Emotion - seine Angst, seinen Ärger, oder was sonst anstehen mag - aufgrund der Zuordnung einer vom Therapeuten konstruierten und von ihm - dem Leidenden - akzeptierten Ursache zurücknimmt und so praktisch das »sozial nicht eingeordnete« Verhalten zugunsten eines »sozial untadeligen ${ }^{21}$ aufgibt. Daß dieser Erfolg - und es ist einer - dem Psychologen Recht gibt, kann nicht behauptet werden. Es ist nämlich durch einen Erfolg dieser Art alles eher denn ausgemachte Sache, daß Ärger oder Angst das sind, als was die Psychotherapie sie traktiert - ein Problem, das der Patient mit sich hat: seine Subjektivität ist mit sich selbst uneins. Im beschriebenen Sinne erfolgreich - d.h. zu vergleichsweiser Harmonie des Individuums mit den Umständen seiner Existenz führend - können Ärger oder Angst nämlich durchaus therapiert werden, wenn sie ihrem Inhalt nach nicht im mindesten gegen das Subjekt des Ärgers oder der Angst, hingegen sehr wohl gegen das, worüber es sich ärgert oder wovor es Angst hat, sprechen, mithin bestens begründet sind.

\section{ANMERKUNGEN}

1) Edmund Husserl: Logische Untersuchungen. Bd. II. Tl, 1. 2. Aufl. Halle: Niemeyer 1913.S. 388.

2) Der Vorgang der Präpositionalisierung, dessen Resultat in einigen Fällen mit der Form des einfachen Objekts koexistiert - njemand fürchtet sich vor erwas * wird neben *jemand fürchtet etwas* gebraucht -, zählt zu den Tendenzen der Gegenwartssprache. Vgl. Peter Braun: Tendenzen in der deutschen Gegenwartssprache. Stuttgart: Kohlhammer 1979. S. 44, 46, 166.

3) Diese Suggestion kann gemindert werden, wenn statt vorn Objekt einer Emotion von ihrer $*$ Richrung* gesprochen wird. S. Ludwig Wittgensrein: Philosophische Untersuchungen $\$ 476$. Werkausgabe. Frankfurt/M.: Suhrkamp 1984. Bd. 1. S. 427.

4) Hunger ist ein Bedürfnis, also nicht einfach ein Gefühl, auch nicht einfach Mangel (Bedarf), sondern Gefühl eines Mangels mit dem Streben, ihn zu beseitigen.

5) Der Grad der Bestimmtheit eines Begehrens kann variieren. Hunger erfor- dert Nahrung - dies ein Titel, unter den sich viele sehr verschiedene Dinge bringen lassen (Georg Wilhelm Friedrich Hegel: Grundlinien der Philosophie des Rechts $₫ 63$. Werke. Hg. v. Eva Moldenhauer u. Karl Markus Michel. Frankfurt/M.: Suhrkamp 1986. Bd. 7. S. 137) -, während der Anblick einer bestimmten Nahrung ein Begehren wecken mag, das nur durch das Essen gerade dieser Nahrung befriedigt werden kann. Im allgemeinen, d.h. solange sich jemand nicht gerade in das Radieschen, das er verspeisen will, verliebt hat, bleibt sie jedoch ersetzbar durch einen anderen Gegenstand der selben Art.

6) David Hume: A Treatise of Human Nature. Hg. v. Lewis Amherst Selby-Bigge. 2. Aufl. Oxford: Clarendon Press 1978. S. 278: „We must, therefore, make a distinction betwixt the cause and the object of the [...] passions; betwixt that idea, which excites them, and that to which they direct their view when excited $\kappa$. Vgl. S. 277, 286f., 304, 305, 329ff., 481f. - Vgl. ferner Wittgenstein: Lectures, Cambridge 1930-1932. Hg. v. Desmond Lee. Totowa, N.J.: Rowman \& Littlefield 1980. S. 112. Ders.: Philosophische Untersuchungen $\$ 476$. Werkausgabe (wie Anm. 3). Bd. 1. S. 426f. Ders.: Zettel $\$ 488$. Ebd. Bd. 8. S. 388.

7) Vgl, die freilich wesentlich abweichende Diskussion desselben Gegenstandes bei Hume: Treatise (wie Anm. 6). S. 277f.

8) Vgl. allerdings Wittgenstein: Philosophische Untersuchungen $\$ 480$. Werkausgabe (wie Anm. 3). Bd. 1. S. 428.

9) Vgl. hierzu Wittgenstein: Zettel $₫ 492$. Werkausgabe (wie Anm. 3). Bd. 8. S. 389.

10) Vgl. z.B. Plato: Timaeus 88a; Denis Diderot: Art, "Passion $\alpha$. In: Dictionnaire Encyclopédique (1778). Oeuvres complètes. Hg. v. Jules Assézat. Bd. XVI. Paris: Garnier 1876. S. 207 - 221. S. 207; Immanuel Kant: Versuch über die Krankheiten des Kopfes. Gesammelte Schriften. Hg. v. d. Königlich Preussischen Akademie der Wissenschaften. Abt. I: Werke. Bd. II. Berlin: Reimer 1905. S. 257 -271. S. 261f., Anthropologie in pragmatischer Hinsicht $\$ \$ 73$ u. 74. Ebd. Bd. VII. 1907. S. 251ff.; Arthur Schopenhauer: Die Welt als Wille und Vorstellung II. Sämtliche Werke. Hg. v. Arthur Hübscher. 4. Aufl. Mannheim: Brockhaus 1988. Bd. 3. S. 681.

11) Wolfgang Schönpflug u. Ute Schönpflug: Psychologie. Allgemeine Psychologie und ihre Verzweigungen in die Entwicklungs-, Persönlichkeits- und Sozialpsychologie. München - Wien - Baltimore: Urban \& Schwarzenberg 1983. S. 414.

12) Sigmund Freud: Vorlesungen zur Einführung in die Psychoanalyse. Gesammelte Werke. Bd. XI. 5. Aufl. Frankfurt/M.: Fischer 1969. S. 411.

13) Schönpflug u. Schönpflug: Psychologie (wie Anm. 11). S. 414.

14) Friedrich Wilhelm Rexrodt: Gehirn und Psyche. Hirnforschung und Psychologie in ihren Zusammenhängen erklärt. Stuttgart: Hippokrates 1981. S. 190.

15) Horst-Eberhard Richter: Der Gotteskomplex. Die Geburt und die Krise des Glaubens an die Allmacht des Menschen. Reinbek: Rowohlt 1979.

16) Freud: Vorlesungen (wie Anm. 12). S. 411.

17) Freud: Die Traumdeutung. Werke (wie Anm. 12). Bd. II/III. 4. Aufl. 1968 S. 406. Zur Logik des "post hoc, ergo propter hoc « vgl. David E. Stannard: Shrinking History. On Freud and the Failure of Psychohistory. Oxford: Oxford University Press 1980. Bes. S. 24, 66, $71 \mathrm{ff}$.

18) Freud: Hemmung, Symptom und Angst. Werke (wie Anm. 12). Bd. XIV. 3. Aufl. 1963. S. 163.

19) Freud: Vorlesungen (wie Anm. 12). S. 454.

20) Freud: Neue Folge der Vorlesungen zur Einführung in die Psychoanalyse. Werke (wie Anm, 12). Bd. XV. 4. Aufl. 1967. S. 85

21) Freud: Bemerkungen über die Übertragungsliebe. Werke (wie Anm. 12). Bd. X. 4. Aufl. 1967. S. 319. 